\title{
Estudio cinemático de la mano para movimiento de oposición del dedo pulgar y movimientos de flexión y extensión de los dedos índice, medio, anular y meñique
}

\section{Hand kinematic study for the thumb opposition movements and the flexion- extension movements of the index, medium, annular and little fingers}

\author{
RUIZ-CHICAIZA, Pablo**i1 \& CEVALLOS-BARRAGÁN, Carlos ${ }^{1,2}$ \\ ${ }^{1}$ Escuela Politécnica Nacional - Facultad de Ingeniería Mecánica - Departamento de Ingeniería Mecánica \\ ${ }^{2}$ LNMB, Université Libre de Bruxelles, Neuroscience Institut, Université Libre de Bruxelles, Brussels, Belgium
}

ID $1^{\mathrm{er}}$ Autor: Pablo, Ruiz-Chicaiza / ORC ID: 0000-0003-2397-1240

ID $1^{\text {er }}$ Coautor: Carlos, Cevallos-Barragán / ORC ID: 0000-0002-4699-670X

DOI: $10.35429 /$ JOIE.2019.11.3.10.21

Recibido 19 de Marzo, 2019; Aceptado 30 de Junio, 2019

\section{Resumen}

El presente estudio analiza la cinemática articular de la mano para el movimiento de oposición del dedo pulgar y los movimientos de flexión y extensión de los dedos. Se utilizó captura de movimiento (MOCAP) con fotogrametría en un laboratorio especializado. Los datos de MOCAP fueron obtenidos con la ubicación de marcadores pasivos en puntos anatómicos de la mano, recomendados por la Sociedad Internacional de Biomecánica (ISB). El estudio se realizó en nueve participantes sin ningún tipo de problema neuronal, reumatológico o traumatológico. Para el análisis de los datos, se utilizó un método de análisis por rotación de juntas tridimensionales. Se demarcaron ciclos estándares para los rangos de movimiento propuestos. Posteriormente, los datos conseguidos fueron alimentados en un modelo virtual mediante el software "OpenSim" para simular los rangos de movimientos obtenidos y corroborar con estudios similares. Se concluye que los rangos de movimiento de cada una de las articulaciones de la mano en los gestos realizados son acordes a estudios similares. Con esto, se permite un posterior estudio de mayor alcance relacionado a la comparación de desórdenes motrices de la mano contra parámetros normales de la población.

Articulación, cinemática, fotogrametría

\begin{abstract}
Present work studies hand articular kinematics for opposition movements of the thumb and flexionextension movements of the fingers. We gathered data by means of Motion capture (MOCAP) photogrammetry and passive markers on the participant hand located in anatomical points recommended by International Society of Biomechanics (ISB). We collected data from nine participants who did not have any neuronal, rheumatologic or traumatological problems. Cycles were demarcated for the study's ranges of movement and analyzed with the rotation of three-dimensional joints method. Subsequently, obtained data was fed in a virtual model in 3D through the software "OpenSim" to simulate the obtained ranges of movement and compared with similar studies. We found that with the proposed methodology, ranges of movement for each hand joint in the performed exercises are acceptable between subjects and according to similar studies. This will allow a later study of greater scope focused in the comparison of people suffering from hand disorders compared to a population standard.
\end{abstract}

Joints, kinematic, photogrammetric

Citación: RUIZ-CHICAIZA, Pablo \& CEVALLOS-BARRAGÁN, Carlos. Estudio cinemático de la mano para movimiento de oposición del dedo pulgar y movimientos de flexión y extensión de los dedos índice, medio, anular y meñique. Revista de Ingeniería Innovativa. 2019. 3-11: 10-21

\footnotetext{
*Correspondencia al Autor (Correo electrónico: pandrer9@gmail.com)

$\dagger$ Investigador contribuyendo como primer Autor.
} 


\section{Introducción}

El campo de estudio de la biomecánica tiene importancia en el desarrollo de métodos y técnicas para el análisis de la fisiología de movimiento dentro del cuerpo humano (Cheron et al., 2016; González, 2019; Tamayo Bullon, 2019). Las diferentes áreas de estudio, como son la antropometría, la cinemática, la cinética y la electromiografía (EMG) ayudan a evaluar de forma específica las características funcionales de movimiento que tiene la mano. Dentro de estas áreas, realizar un estudio del movimiento de las articulaciones (cinemática articular) permite obtener estándares para validar el movimiento de objetos robóticos y comparación de movimientos en estudios biomédicos (Conti, Meli, y Ridolfi, 2016; Davis, Ounpuu, Tyburski, y Gage, 1991; Lee y Jung, 2015).

El área de estudio de la cinemática de la mano se enfoca en evaluar ángulos, trayectorias, velocidades y las aceleraciones, a partir de los gestos funcionales. Existen técnicas que facilitan la evaluación del movimiento cinemático, no obstante, cada una cuenta con distintos alcances. Algunas de las técnicas utilizadas para el estudio cinemático de la mano son: rayos-X, imágenes por resonancia magnética, goniómetros manuales, electro goniometría, video análisis, y marcadores basados en sistemas de análisis de movimiento (Lee y Jung, 2015).

En el trabajo de Baker et al. (2007) se realizó el cálculo de las posturas de la muñeca y los dedos de la mano, en el que comparó las técnicas de goniometría y capturas de movimiento, donde resalta la dificultad de identificar movimientos complejos de la mano, mientras que el uso de captura de movimiento facilita la adquisición de los movimientos complejos de la mano.

Coupier et al. (2016) analizó los movimientos de flexión-extensión y aducciónabducción de los dedos de la mano mediante fotogrametría, donde señaló la importancia de la calibración de las cámaras y ubicación de los marcadores en las manos.
Conti et al. (2016) construyó un exoesqueleto de arquitectura portable, en su investigación realizó un modelo cinemático de la mano y lo validó mediante la comparación entre el modelo cinemático y la captura del movimiento de la mano. Coert et al. (2003) cuantificó el movimiento circundante del pulgar mediante técnica de video. En su investigación resaltó la importancia de estudiar los movimientos del pulgar ya que es un movimiento esencial para realizar las funciones normales de la mano.

Kapandji (2006) enfatiza que los movimientos más importantes en relación con su funcionalidad son: el movimiento de flexión y extensión de los dedos, además de la oposición del dedo pulgar hacia los dedos trifalángicos (DTF). Nakamura et al. (1999) analizó en vivo la rotación normal del brazo utilizando un método de resonancia magnética, en esta técnica señala que además de utilizar resonancia magnética era necesario otros instrumentos como el goniómetro para realizar el cálculo.

Mallon et al. (1991) utiliza la técnica de electro goniometría (uso de goniómetros digitales) para evaluar el rango de movimiento (ROM) en las articulaciones de las manos de personas saludables, donde enfatiza que no existe diferencias notables al realizar la comparación de rangos dependiendo del sexo. Batmanabane y Malathi (1985) utiliza la técnica de rayos $\mathrm{X}$ para evaluar los ROM de los ejercicios de aducción/abducción en los DTF. Una vez obtenida la radiografía, ubica los huesos evaluados y mediante dos líneas calculó el ángulo generado.

Con base en las facilidades y dificultades mostradas previamente en el párrafo anterior, la presente investigación tiene como objetivo el estudio cinemático articular de la mano para los movimientos de oposición del dedo pulgar hacia los DTF y la flexión y extensión de los dedos. Mediante la técnica de fotogrametría (MOCAP) se logrará obtener las trayectorias que sigue cada uno de los dedos acorde con los movimientos señalados. El aporte de esta investigación en la biomecánica, con base en lo anterior mencionado, será ayudar a obtener modelos cinemáticos estándares de la mano realizando movimientos combinados entre el dedo pulgar y los DTF. 
Además, al ser parte del proyecto de vinculación PVS 2017011 se logra crear relaciones de trabajo entre diferentes proyectos de titulación y gracias a la colaboración de BioFoot y KINÉTICO, entidades dedicadas al tratamiento personas en procesos de rehabilitación física, el presente trabajo se proyecta como punto de partida para nuevas investigaciones en el Ecuador.

\section{Metodología a desarrollar}

La presente investigación pasó por un proceso de aprobación de protocolo de estudios previo avalado por el Comité de Ética de la Universidad Católica del Ecuador, en el cual, se garantiza que todos los procesos se realizarán con seguridad para el participante de acuerdo a la Declaración de Helsinki y se respetarán los datos personales de los mismos (Código 2018-29-EO). Los movimientos principales para estudiar son los siguientes:

\section{- $\quad$ Movimientos de flexión-extensión de los DTF, comenzando con flexión. \\ - Movimientos de oposición del dedo pulgar hacia los DTF, comenzando con el índice hasta terminar con el meñique.}

Mejía (2000) enfatiza que, el muestreo por conveniencia puede ser utilizado para definir hipótesis, establecer nuevas características a la investigación y servir como punto de partida en líneas de investigación. Otzen y Manterola (2017) relatan las ventajas de utilizar este tipo de muestreo no probabilístico, en el que se tiene mayor accesibilidad y proximidad al tipo de muestra que uno desee estudiar.

Por lo tanto, la presente investigación, al ser una de las primeras enfocadas en utilizar técnicas como la fotogrametría en el Ecuador, se decidió utilizar un muestreo no probabilístico como lo es el muestreo por conveniencia, en el cual, se tomó una muestra de 9 personas (5 hombres y 4 mujeres) en un rango de edad de 18 a 30 años (media 24 años) Las personas que participaron en este estudio no debían tener ningún tipo de problema neuronal o físico. Las grabaciones se hicieron con un sistema de fotogrametría (BTS Bioengineering) calibrado y equipado con 6 cámaras optoelectrónicas de alta velocidad $(70 \mathrm{~Hz})$ y 2 cámaras análogas para control $(30 \mathrm{~Hz})$. La participación fue voluntaria y sin ningún tipo de remuneración.
Posterior a la toma de datos, firmaron un documento de consentimiento informado, en el que consta todos los detalles de cómo se utilizarán sus datos y los derechos que tiene la persona. Estos datos para la muestra, adquiridos de cada una de las personas, se los obtuvo a partir de un protocolo de pruebas. El participante sigue este procedimiento hasta la culminación de la adquisición.

Cada participante es informado de los procedimientos a seguir en el laboratorio, dentro de los cuales se siguieron estos pasos:

1. Se proporcionó información sobre los objetivos que se pretende alcanzar mediante la adquisición de datos, la importancia de los trabajos de investigación, número de sesiones a realizar, el procedimiento que se seguirá, el compromiso entre el participante y el investigador.

2. Se realizó un cuestionario para saber si el participante se encuentra en las condiciones adecuadas para realizar los procedimientos requeridos. En esta encuesta también se incluyen algunos datos importantes (edad, género).

3. Una vez que se verificó mediante el cuestionario que el participante puede realizar los procedimientos requeridos, se procede a hacer firmar al participante un consentimiento informado. El consentimiento informado proporcionó al participante información sobre sus derechos y expresa la decisión voluntaria de participar en el estudio.

Para cada uno de los ejercicios que se van a realizar se ubicaron marcadores reflectivos pasivos en la mano del participante con cinta hipoalergénica siguiendo el sistema utilizado por la Sociedad Internacional de Biomecánica (Wu et al., 2005), los puntos fueron ubicados acorde con los siguientes ejercicios:

Para el ejercicio de flexión-extensión de los DTF se utilizó dos configuraciones de ubicación: 
a. En la primera configuración se utilizaron 12 marcadores: 4 marcadores en una base a $3 \mathrm{~cm}$ de la muñeca, Figura 1A; 4 marcadores en el dedo índice, 3 de los 4 marcadores ubicados en el medio de cada falange y el restante $3 \mathrm{~cm}$ atrás de la junta metacarpofalángica, en el hueso metacarpiano del índice; y los siguientes 4 marcadores se ubican de la misma forma que los 4 marcadores ubicados en el dedo índice pero aplicados al dedo anular.

b. En la segunda configuración se utilizó 12 marcadores, ubicados en la misma distribución, pero para los dedos medio y meñique, Figura 1B.

Para el ejercicio de oposición del dedo pulgar se utilizó dos configuraciones de ubicación:

a. En la primera configuración se utilizaron 15 marcadores: 4 marcadores en una base a $3 \mathrm{~cm}$ de la muñeca, Figura 1C; 4 marcadores en el dedo índice, 3 de los 4 marcadores ubicados en el medio de cada falange y el restante se ubica $3 \mathrm{~cm}$ atrás de la junta metacarpofalángica, en el hueso metacarpiano del índice; los siguientes 4 marcadores de la misma forma que los marcadores ubicados en el dedo índice pero aplicados al dedo anular; y los últimos 3 marcadores en el medio de cada una de las falanges del dedo pulgar y en el medio del hueso metacarpiano del pulgar.

b. En la segunda configuración se utilizaron 15 marcadores, ubicados en la misma distribución, pero para los dedos medio y meñique, mientras que, el pulgar permanece con la misma configuración, Figura 1D.

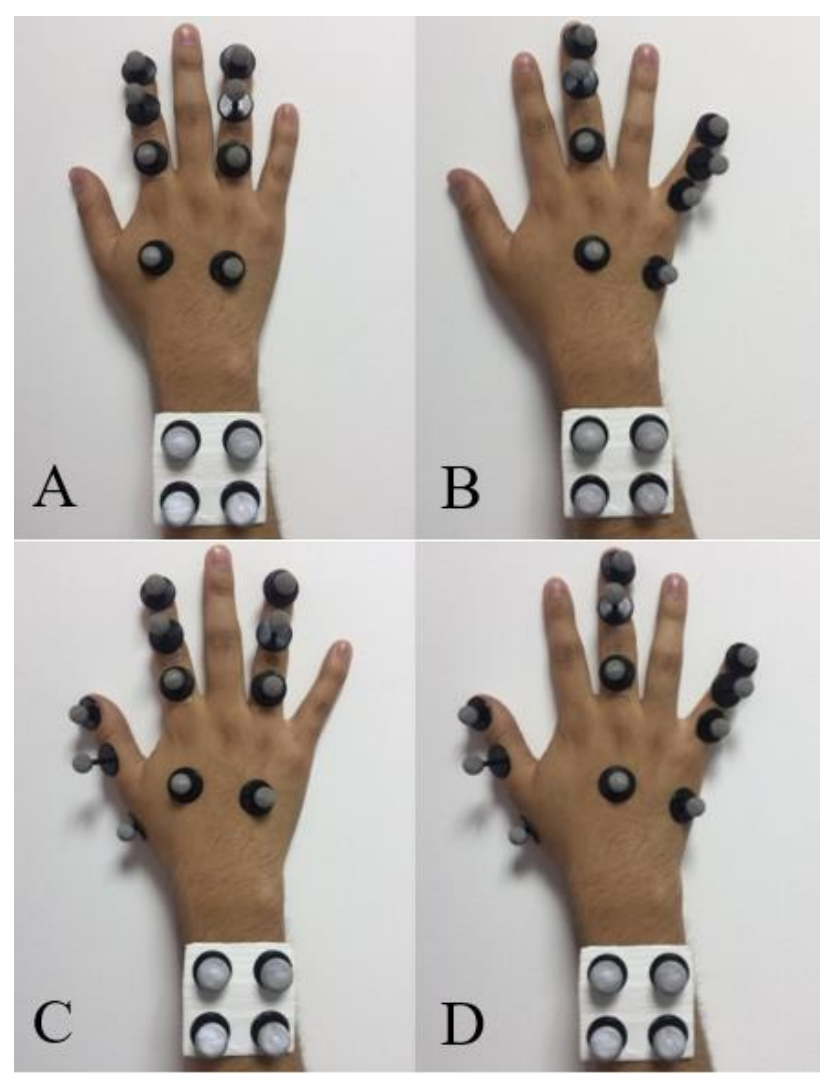

Figura 1 Posicionamiento de marcadores para los ejercicios realizados por la mano. A y B. Flexiónextensión. C y D. Oposición del dedo pulgar

Fuente: Elaboración Propia

Se tomo como referencia para el cálculo de ROM de los ejercicios de flexión/extensión de los DTF y oposición del dedo pulgar al método realizado por Cheng y Pearcy (1999). Sin embargo, el cambio realizado de este método es la utilización de un sistema de coordenadas móvil para anular la relación que existe entre los movimientos de flexión/extensión y abducción/abducción, La Figura 2 ilustra el sistema de coordenadas móvil que reduce un error mínimo $\left(1-1.75^{\circ}\right)$ en el valor del ángulo de flexión y extensión generado al tener un sistema de coordenadas fijo.

Una vez que se obtuvieron los modelos cinemáticos de cada dedo para los ejercicios realizados, se exportaron los modelos cinemáticos en el modelo CAD desarrollado por Gonzalez et al. (1997) utilizado en la biomecánica en la herramienta "Opensim" (Delp et al., 2007) para observar si cumplen los resultados con lo esperado. 


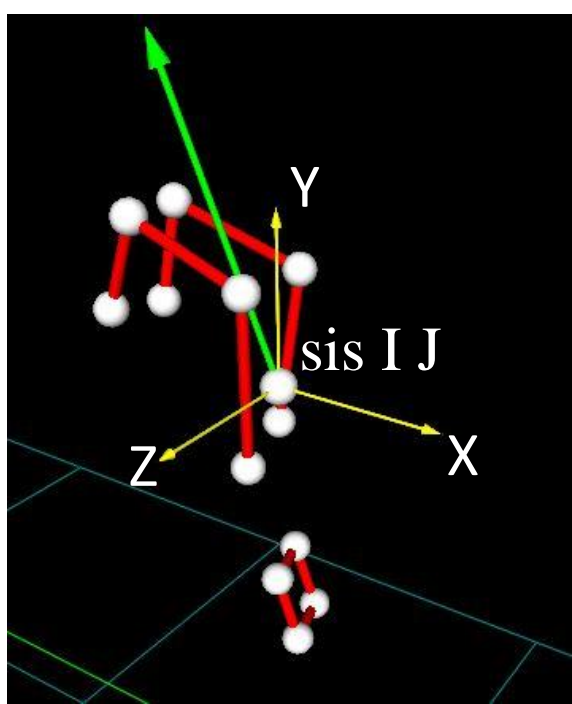

Figura 2 Sistema de coordenadas móvil para el cálculo de ángulo generado en flexión/extensión del vector unitario. En verde se visualiza el vector unitario. En amarillo se observa el sistema de coordenadas

Fuente: Elaboración Propia

\section{Resultados}

Los resultados de cinemática articular de los DTF (II, III, IV, V) durante movimientos de flexión y extensión se encuentran detallados en la Tabla 1.

La articulación con el mayor ángulo de flexión-extensión es la articulación metacarpofalángica del dedo $\mathrm{V}\left({ }^{\mathrm{f}}\right) \operatorname{con} 81^{\circ}$ y una desviación estándar de $21^{\circ}$. Mientras que, la articulación en el menor ROM es la articulación interfalángica distal del dedo $\mathrm{V}\left({ }^{(}\right) \operatorname{con} 61^{\circ}$ y una desviación estándar de $14^{\circ}$. Una de las causas del incremento de la desviación estándar se produjo debido que los participantes no realizaron adecuadamente los movimientos pese a haber sido informados de los movimientos que debían realizar.

Además, los marcadores respecto a los dedos son bastante grandes e incómodos, lo cual ocasiona en ciertos casos que los participantes no realicen el movimiento adecuado de los dedos. Por lo tanto, la desviación estándar sirve como rangos en los cuales una persona puede tener un movimiento normal de la mano.

En el Gráfico 1A se ilustra el ROM de las juntas metacarpofalángicas de cada uno de los DTF.
En esta gráfica se observa que el mayor ROM respecto a las articulaciones metacarpofalángicas se da en el dedo V (verde), mientras que el menor ROM en el dedo II (negro) y sin embargo, se debe resaltar que dedo III (rojo) cuenta con un movimiento atípico al tener dos picos de movimiento (flechas A), esto se debe a que sobrepasó el límite de 0 a $\pi$ proporcionado por las ecuaciones de Cheng y Pearcy y no se logró obtener el punto máximo de movimiento, aunque si se puede observar que su pico máximo si se encuentra relacionado con el movimiento normal de los dedos.

En el Gráfico 1B se ilustra el ROM de las juntas interfalángicas proximales de los DTF donde se visualiza que todos los movimientos tienen un comportamiento de flexión-extensión normal, en el cual, con un mayor ROM llega el dedo III (rojo). Sin embargo, se debe resaltar que el dedo IV (azul) tiene un movimiento negativo que nos indica una hiperextensión en el dedo IV (azul). También se observa que el dedo III (rojo) y V (verde) duran más tiempo en la fase de flexión, al contrario de los dedos II (negro) y IV (verde) en los cuales, dura menos.

\begin{tabular}{|l|l|r|r|}
\hline Dedos & Articulaciones & \multicolumn{1}{r|}{ Flex/Ext } & DS \\
\hline D II & MCF & 62 & 27 \\
\hline & IFP & 73 & 16 \\
\hline & IFD & 77 & 16 \\
\hline D III & MCF & 67 & 22 \\
\hline & IFP & 78 & 12 \\
\hline & IFD & 69 & 13 \\
\hline D IV & MCF & 76 & 26 \\
\hline & IFP & 80 & 14 \\
\hline & IFD & 71 & 20 \\
\hline D V $^{f_{f}}$ & MCF & $81^{\mathrm{f}}$ & $21^{\mathrm{f}}$ \\
\hline & IFP & 75 & 12 \\
\hline & IFD $^{\text {f }}$ & $61^{\mathrm{f}}$ & $14^{\mathrm{f}}$ \\
\hline
\end{tabular}

Tabla 1. Media del ROM y desviación estándar de las articulaciones durante los movimientos de flexión y extensión de los DTF

Fuente: Elaboración Propia

En el Gráfico 1C se puede apreciar el ROM de las juntas interfalángicas distales de los DTF, donde se visualiza que el dedo II (negro) cuenta con el mayor ROM, sin embargo, también se observa el mismo fenómeno que en el Gráfico 1A en la que se observan dos picos debido a que salen del rango, dedo III (rojo A) y $\mathrm{V}$ (verde B). 
Esto quiere decir que, si bien es cierto que el dedo II (negro) obtienen el mayor ROM, se deben considerar los dedos III (rojo) y V (verde) ya que no se observa su pico máximo de movimiento. Inclusive el rango del dedo III (rojo) puede llegar a ser igual o mayor que el rango del dedo II (negro).
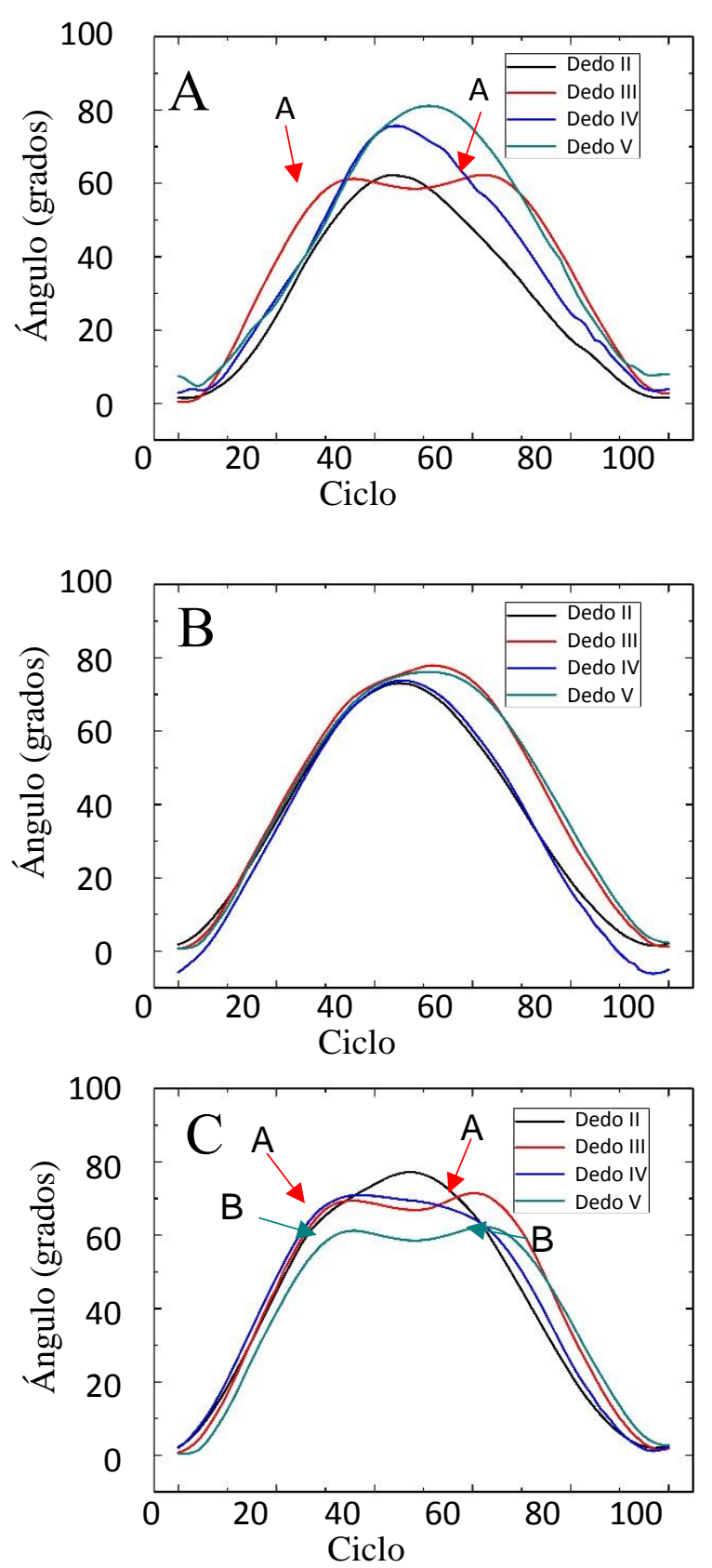

Gráfico 1. Movimiento de flexión-extensión de las articulaciones: A. metacarpofalángicas de los DTF. B. Interfalángicas proximales de los DTF. C. Interfalángicas distales de los DTF. Ángulo $0^{\circ}$ representa la posición de extensión normal de la mano o punto inicial del movimiento de flexión, llegado al punto máximo en la mitad del ciclo comienza la extensión

Fuente: Elaboración Propia
En la Tabla 2 se presentan los resultados de cinemática articular del dedo pulgar hacia los DTF (pinza terminal). Los ROM de las articulaciones de los DTF para cada uno de los ejercicios de oposición del dedo pulgar hacia los DTF (índice, medio, anular y meñique) junto con sus desviaciones estándar.

\begin{tabular}{|c|c|c|c|c|c|c|c|}
\hline Dedo & Articulación & $\mathrm{FE}$ & DS & $\begin{array}{l}\mathrm{Ad} / \\
\mathrm{Ab}\end{array}$ & DS & $\begin{array}{c}\text { Min/ } \\
\text { Max FE }\end{array}$ & $\begin{array}{c}\mathrm{Min} / \\
\mathrm{Max} \\
\mathrm{Ad} / \\
\mathrm{Ab}\end{array}$ \\
\hline \multicolumn{8}{|c|}{ Dedos índice y pulgar } \\
\hline \multirow[t]{3}{*}{ DI } & CMC & 7 & 3 & 13 & 5 & $1-7$ & $1-13$ \\
\hline & MCF & 17 & 13 & & & $1-17$ & \\
\hline & IF & 52 & 29 & & & $8-52$ & \\
\hline \multirow[t]{3}{*}{ DII } & MCF & 30 & 17 & & & $1-30$ & \\
\hline & IFP & 24 & 11 & & & $45-69$ & \\
\hline & IFD & 25 & 12 & & & $30-55$ & \\
\hline \multicolumn{8}{|c|}{ Dedos medio y pulgar } \\
\hline \multirow[t]{3}{*}{$\mathrm{DI}$} & CMC & 9 & 4 & 16 & 8 & $2-9$ & $2-16$ \\
\hline & MCF & 16 & 14 & & & $1-16$ & \\
\hline & IF & 63 & 60 & & & $3-63$ & \\
\hline \multirow[t]{3}{*}{ DIII } & $\mathrm{MCF}$ & 20 & 10 & & & $1-20$ & \\
\hline & IFP & 29 & 9 & & & $46-74$ & \\
\hline & IFD & 29 & 11 & & & $46-74$ & \\
\hline \multicolumn{8}{|c|}{ Dedos anular y pulgar } \\
\hline \multirow[t]{3}{*}{ DI } & $\mathrm{CMC}$ & 10 & 6 & 20 & 9 & $1-10$ & $1-20$ \\
\hline & $\mathrm{MCF}$ & 19 & 18 & & & $2-19$ & \\
\hline & IF & 90 & 93 & & & $5-90$ & \\
\hline \multirow[t]{3}{*}{ DIV } & MCF & 34 & 13 & & & $2-34$ & \\
\hline & IFP & 38 & 12 & & & $46-83$ & \\
\hline & IFD & 48 & 14 & & & $32-78$ & \\
\hline \multicolumn{8}{|c|}{ Dedos meñique y pulgar } \\
\hline \multirow[t]{3}{*}{ DI } & CMC & 14 & 9 & 22 & 12 & $2-14$ & $1-22$ \\
\hline & $\mathrm{MCF}$ & 21 & 21 & & & $4-21$ & \\
\hline & IF & 64 & 44 & & & 6-64 & \\
\hline \multirow[t]{3}{*}{ DV } & MCF & 46 & 17 & & & $1-46$ & \\
\hline & IFP & 46 & 26 & & & $1-46$ & \\
\hline & IFD & 52 & 16 & & & $33-82$ & \\
\hline
\end{tabular}

Tabla 2 ROM de las articulaciones del dedo anular y pulgar para movimiento de oposición en conjunto con la desviación estándar (DS). Flexión / Extensión (FE) Los datos son medidos en grados $\left(^{\circ}\right)$

Fuente: Elaboración Propia

El Gráfico 2A representa el ROM de la junta carpometacarpiana del dedo pulgar y su movimiento hacia los DTF. Como se observa, el dedo con mayor ROM es el dedo V (verde), mientras que, el dedo II (negro) obtiene el mayor ROM debido a que se encuentra más alejado del dedo pulgar el dedo meñique. Se debe notar que en el dedo IV (azul) se tiene ruido en el movimiento (A), esto puede darse debido a que los puntos se llegan a ocultar de las cámaras y no se logra capturar el movimiento.

En el Gráfico 2B se observa el ROM en aducción-abducción de la junta carpometacarpiana del dedo pulgar en relación con los movimientos de oposición del dedo pulgar.

RUIZ-CHICAIZA, Pablo \& CEVALlOS-BARRAGÁN, Carlos. Estudio cinemático de la mano para movimiento de oposición del dedo pulgar y movimientos de flexión y extensión de los dedos índice, medio, anular y meñique. Revista de Ingeniería Innovativa. 2019. 
En esta se puede apreciar que no lleva ningún tipo de ruido en sus movimientos, además que el dedo con llevar el mayor ROM es el dedo V (verde), mientras que, el dedo II (negro) realiza el menor movimiento respecto a los demás dedos. Se debe tomar en cuenta que los ángulos tomados siguen con la lógica de a una mayor distancia de los dedos hacia el pulgar, realizan un mayor ROM.

El Gráfico 2C representa el movimiento de flexión extensión de la articulación metacarpofalángica del dedo pulgar. En esta gráfica se puede observar que el mayor movimiento de aducción-abducción lo realiza el dedo $\mathrm{V}$ (verde), mientras que el dedo con el menor ROM es el dedo III (rojo). Este caso se debe a que el dedo más grande de la mano es el dedo medio III (rojo) y el pulgar debe trasladarse menos que en el caso de los demás dedos. Se debe notar que las gráficas no se mantienen con la misma geometría, sin embargo, mantienen su movimiento suave y no uno brusco. En este gráfico se observa que no hay mucho ruido que hubiera afectado a la captura de movimiento.

En el Gráfico 2D se observa los movimientos de flexión y extensión de la articulación interfalángica del dedo pulgar hacia los DTF. En esta gráfica se puede apreciar que se tiene varios puntos de ruido en todas las gráficas de los dedos, con excepción del dedo $\mathrm{V}$ (verde), en el cual, se conserva un movimiento bastante suave respecto a los demás. Principalmente existe más ruido en los puntos iniciales (A rojo) y finales (B azul), que vienen a ser los puntos de extensión de los dedos. La causa general para que se realicen este tipo de ruidos se da por los puntos que se ocultan de las cámaras y no logran ser identificados para realizar su respectivo rastreo o tracking.

En el Gráfico 3A se puede observar el movimiento de flexión extensión de los DTF en su junta metacarpofalángica para los ejercicios de oposición del dedo pulgar. En esta gráfica se puede observar que nuevamente el dedo con el mayor ROM es el V (verde), mientras que el dedo con un menor ROM es el dedo III (rojo). También se presentan ruido en los movimientos iniciales del dedo $\mathrm{V}$ (verde $\mathrm{A}$ ) y finales del dedo IV (azul B).
Estos movimientos se repiten para las articulaciones interfalángicas proximales y distales debido a que se genera un mayor movimiento a medida que se encuentran los dedos alejados del pulgar, como se observa en los Gráficos 3By 3C. En el caso de la junta interfalángica proximal (Gráfico 3B) el que realiza el menor movimiento es el dedo III (rojo) mientras que el dedo $\mathrm{V}$ (verde) continúa realizando el mayor ROM, además, se observa ruido en el dedo II (negro A) al final del ciclo. Observando el movimiento de la articulación interfalángica distal (Gráficos 3C) el que realiza el menor movimiento es el dedo II (negro) y el dedo V (verde) realiza el mayor movimiento, sin embargo, el dedo III (rojo) no se aleja mucho del movimiento del dedo II (negro), por lo que se sigue manteniendo la tendencia de ser el que realiza menos movimiento hacia el dedo pulgar.

Al momento de realizar el rastreo "tracking" de los movimientos capturados se tuvo problemas en los ejercicios de oposición del dedo pulgar ya que al tener un mayor número de marcadores se generó el fenómeno de oclusión, para el caso de la flexión/extensión de los DTF no se tuvo mayor problema ya que los movimientos realizados son más simples y con menor número de marcadores.

El protocolo de prueba para la adquisición de movimientos cinemáticos tomó en cuenta que únicamente se puede realizar para una persona a la vez y no de forma colectiva, esto se produjo ya que existieron datos iniciales que ingresaron en el protocolo para que se realice el cálculo. Además, se debe tomar en cuenta que todos los rangos calculados de las articulaciones de los dedos se realizaron a partir de sistemas de referencia ubicados para el caso de los DTF en la J MCP y para el caso del dedo pulgar ubicado en la J TMC, a causa de que los marcadores fueron ubicados en los puntos medios de cada falange. Por consiguiente, no se pudo realizar la creación de las demás articulaciones, por lo que se optó por esta opción. 

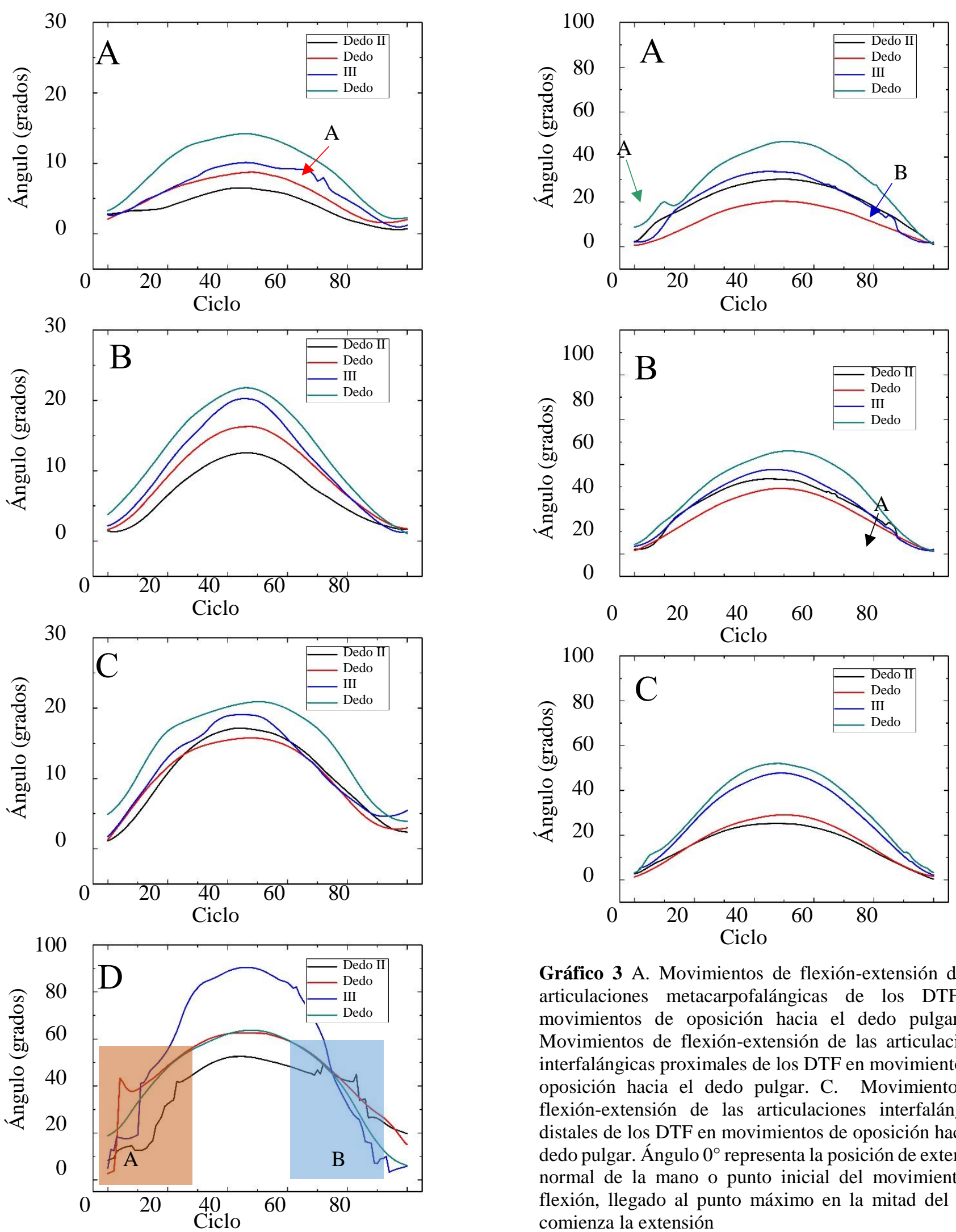

Gráfico 2 A. Movimientos de flexión-extensión de la articulación carpometacarpiana del dedo pulgar hacia los DTF. B. Movimientos de aducción-abducción de la articulación carpometacarpiana del dedo pulgar hacia los DTF. C. Movimientos de flexión-extensión de la articulación metacarpofalángica del dedo pulgar hacia los DTF. D. Movimientos de flexión-extensión de la articulación interfalángica del dedo pulgar hacia los DTF. Ángulo $0^{\circ}$ representa la posición de extensión normal de la mano o punto inicial del movimiento de flexión, llegado al punto máximo en la mitad de ciclo comienza la extensión

Fuente: Elaboración Propia

ISSN 2523-6873

ECORFAN ${ }^{\circledR}$ Todos los derechos reservados.

Gráfico 3 A. Movimientos de flexión-extensión de las articulaciones metacarpofalángicas de los DTF en movimientos de oposición hacia el dedo pulgar. B. Movimientos de flexión-extensión de las articulaciones interfalángicas proximales de los DTF en movimientos de oposición hacia el dedo pulgar. C. Movimientos de flexión-extensión de las articulaciones interfalángicas distales de los DTF en movimientos de oposición hacia el dedo pulgar. Ángulo $0^{\circ}$ representa la posición de extensión normal de la mano o punto inicial del movimiento de flexión, llegado al punto máximo en la mitad del ciclo comienza la extensión

Fuente: Elaboración Propia

\section{Análisis de resultados}

La Tabla 1 muestra la media y la desviación estándar de los DTF (DII, DIII, DIV y DV) de la mano derecha durante movimientos de flexión y extensión. Estos datos son menores comparados con los datos obtenidos por (Conti et al., 2016; Degeorges et al., 2005; Mallon et al., 1991; Nordin et al., 2004).

RUIZ-CHICAIZA, Pablo \& CEVALlOS-BARRAGÁN, Carlos Estudio cinemático de la mano para movimiento de oposición del dedo pulgar y movimientos de flexión y extensión de los dedos índice, medio, anular y meñique. Revista de Ingeniería Innovativa. 2019. 
Esta diferencia de resultados se produjo posiblemente a que no se realizaron los movimientos hasta la máxima flexión y extensión de los dedos, al contrario, los movimientos se ejecutaron hasta donde se sienta cómodo el participante.

Coupier et al. (2016) al realizar el análisis cinemático de la mano para movimientos de flexo-extensión, aducciónabducción y pronación-supinación obtiene ROM del dedo pulgar y de los DTF mediante una distribución de marcadores tipo cluster. En la presente investigación se utilizó únicamente un marcador por cada falange ubicado en los puntos recomendados por la Sociedad Internacional de Biomecánica (Wu et al., 2005).

Mediante la distribución propuesta, se obtuvo en la junta metacarpofalángica y en la interfalángica distal, ángulos que se ubican entre los rangos encontrados por Coupier. Únicamente en el caso de la junta interfalángica proximal se obtuvo un ángulo menor por 11 grados.

Mallon et al. (1991) utilizó la técnica de goniometría mediante un goniómetro digital, en la cual, obtuvo ROM mayores que la presente investigación. Únicamente se obtuvo valores similares en la junta interfalángica distal con $77^{\circ}$. Si bien es cierto en los dos estudios no se limita el movimiento para cada participante, se obtienen diferentes resultados ya que no se utiliza la misma técnica de recolección de datos. En la que Mallon et al. realizó la recolección de datos mediante goniometría y la presente investigación mediante MOCAP.

Degeorges et al. (2005) realizó el estudio de las rotaciones realizadas en las juntas de los dedos de la mano mediante una distribución de tres marcadores en cada una de las falanges para obtener sistemas de coordenadas móviles y hallar los ángulos de cada junta. En el presente trabajo no se tiene esta disposición y los ángulos obtenidos son de las proyecciones ortogonales de un vector unitario en los planos del sistema de coordenadas generado. Sin embargo, se obtuvo para todas las juntas de los DTF los ROM que se encuentran en los límites denotados por Degeorges et al. en su publicación.
Otra de las diferencias para la adquisición de datos y la literatura consultada es la forma de capturar el movimiento y su cálculo. Coupier et al. (2016) utilizó sistemas de coordenadas realizados a partir de marcadores ubicados en cada falange, mientras que, en el presente trabajo se utilizaron los marcadores en los puntos medios de las falanges.

Cheng y Pearcy (1999) realizó un modelo para el cálculo de los ángulos generados en las articulaciones, en el cual, utilizó sistemas de coordenadas que permanecen estáticos. En el presente trabajo se utilizaron sistemas de coordenadas que rotan alrededor del eje $\mathrm{X}$ para seguir al movimiento de abducción/abducción generado por las falanges, con lo que se pudo obtener el rango total de flexión/extensión.

La mayor desviación estándar, con un valor de $27^{\circ}$, se da en la articulación MCF (II) y es la de mayor dispersión de datos. En los movimientos de las juntas MFC (III), IFP (III) e IFP (V) no se alcanza a obtener el punto máximo de movimiento, esto se debe a que el sistema de coordenadas para todos los participantes es constante en el eje $\mathrm{X}$ (solo rota), por lo que si una persona ubica la mano en una posición diferente a la indicada, el ángulo formado desde el eje $X$ en el plano XY, Figura 2, superaba la condición dada en la ecuación 3 y tomaba los datos como si estuviera reduciendo su ROM . Por lo que, para el caso de las juntas antes mencionadas, no tienen un punto máximo de movimiento como las otras gráficas. Para el caso de todas las juntas IFP, todos los ROM se conservaron semejantes a diferencia de las juntas MCF e IFD, debido a que su rango de movimiento si se conservaba en los cuadrantes indicados para el movimiento.

Para el movimiento de oposición del dedo pulgar se dividieron los resultados dependiendo del dedo al cual se oponía el dedo pulgar. Para el primer movimiento, Tabla 2 (oposición pulgar - índice), se obtuvo el mayor ángulo de flexión en la junta IF con $52^{\circ}\left(29^{\circ}\right.$ DS), mientras que, el mínimo movimiento de flexión lo realizó la junta $\mathrm{CMC}$ con $7^{\circ}\left(3^{\circ} \mathrm{DS}\right)$; para el segundo movimiento, Tabla 2 (oposición pulgar - medio), el mayor ángulo de flexión se realizó en la junta IF con 63 ( $\left.60^{\circ} \mathrm{DS}\right)$ y el menor ángulo de flexión en la junta $\mathrm{CMC}$ con $9^{\circ}\left(4^{\circ}\right.$ DS); en el tercer movimientos. 
Tabla 2 (oposición pulgar - anular), el mayor ángulo se obtuvo en la junta IF con 90 ( $\left.93^{\circ} \mathrm{DS}\right)$, mientras que el menos ángulo en la junta CMC $10^{\circ}\left(6^{\circ} \mathrm{DS}\right)$; finalmente en el cuarto movimiento, Tabla 2 (oposición pulgar meñique), el ángulo mayor se obtuvo en la junta IF con $64^{\circ}\left(44^{\circ} \mathrm{DS}\right)$ y el menor ángulo en la junta $\mathrm{CMC}$ con $14^{\circ}$ (9 DS).

Como se observa en los resultados las desviaciones estándar en la junta IF son altas debido a que cada persona realiza sus movimientos de diferente forma, además de ser mayores sus ROM que el movimiento realizado por las juntas de los DTF. Se debe resaltar que para los DTF no se calculó la aducción/abducción ya que únicamente el alcance del desarrollo del exoesqueleto en el proyecto de vinculación PVS 2017011 se realiza movimientos de flexión/extensión y con libertad de movimiento de aducción/abducción en los DTF (II, III, IV, V).

En el caso del dedo pulgar al tener un movimiento combinado se necesita conocer también la aducción/abducción. Este ejercicio obtuvo mayor rango en la oposición del dedo pulgar hacia el dedo meñique con ángulo de $22^{\circ}$ (12 $\left.{ }^{\circ} \mathrm{DS}\right)$, Tabla 2 (oposición pulgar - meñique). Esta desviación estándar es considerable con respecto a su media, y se puede apreciar que las curvas de aducción/abducción tienen una tendencia creciente a medida avanzan de dedos DII, DIII, DIV y DV, Gráfico 2B.

No se encontró en la literatura, trabajos en los que se realicen movimientos combinados de oposición del dedo pulgar, sin embargo, una vez ingresados los datos en el software "OpenSim (Delp et al., 2007)" se puede observar que las falanges distales del dedo pulgar y de los DTF se acercan notablemente. Se debe tomar en cuenta que en el modelo utilizado únicamente incluye el hueso de la mano y no los músculos ni la piel de los dedos.

Una vez obtenidos los modelos cinemáticos se implementaron en el modelo CAD de la mano utilizado en este trabajo, desarrollado por Gonzalez et al. (1997) en software "OpenSim (Delp et al., 2007)". En los primeros resultados cinemáticos probados fueron los ángulos de flexión/extensión de los DTF, se aprecia que cumple con los movimientos esperados.
En el caso de la oposición del dedo pulgar se incluyó todos los datos de la oposición del pulgar hacia los demás dedos en un solo archivo. Estos datos tuvieron un menor acercamiento al resultado esperado ya que los dedos no llegaban a cerrar la pinza terminal, sin embargo, se logró un resultado aproximado. Para mejorar los resultados obtenidos en las pruebas realizadas, el protocolo queda abierto para ingresar un mayor número de personar y de esta manera mejorarlo. Todos los datos utilizados se encuentran el en siguiente repositorio digital: https://figshare.com/s/85e3106637b9a5ec2d0e

\section{Agradecimientos}

El presente trabajo fue respaldado por la Escuela Politécnica Nacional - Facultad de Ingeniería Mecánica. Agradecemos por la excelencia académica brindada por la universidad. BIOFOOT, laboratorio de análisis de movimiento. KINETICO rehabilitación por su ayuda en los protocolos y selección de participantes.

\section{Conclusiones}

La presente investigación estableció un estándar cinemático para los movimientos de la mano en personas sin desórdenes motorices de la mano. Los movimientos estudiados de oposición del dedo pulgar y flexión y extensión de los DTF a partir de los modelos cinemáticos son obtenidos en conjunto de sus desviaciones estándar que sirven como un primer acercamiento a estándares de un movimiento normal de la mano.

Se obtuvo modelos cinemáticos, probados en simulación con modelo virtual que pueden ser utilizados en el diseño de prótesis como guías para el movimiento natural de la mano. Los movimientos máximos obtenidos en las articulaciones fueron: MCF $82^{\circ}$, IFP $80^{\circ} \mathrm{e}$ IFD $77^{\circ}$ en los ejercicios de flexión y extensión de los DTF que es similar a la revisión de estudios utilizados para este trabajo (Conti et al., 2016; Degeorges et al., 2005; Mallon et al., 1991; Nordin et al., 2004).

De igual manera, por la similitud de resultados con otras metodologías, se validan tanto el protocolo utilizado para la recolección de datos junto con los movimientos analizados en el presente estudio. 


\section{Trabajos futuros}

La media obtenida, puede ser comparativa para personas en procesos de recuperación del movimiento de la mano como se ha utilizado en dispositivos para rehabilitación motriz (Bonilla Barrero, Pavajeau Escorcía, y Pérez Barbosa, 2019; Gonzalez Cruz, 2019; Zambrano et al., 2018).

La presente investigación con la implementación de sistemas de coordenadas móviles y estándares cinemáticos logra ser un aporte importante dentro del campo de la biomecánica de la mano y su continuidad con su estudio dinámico.

\section{Referencias}

Baker, N. A., Cook, J. R., Baker, N. A., Cham, R., Hale, E., y Redfern, M. S. (2007). Measurements of Wrist and Finger Postures : A Comparison of Goniometric and Motion Capture Techniques Measurements of Wrist and Finger Postures : A Comparison of Goniometric and Motion Capture Techniques. (Septiembre 2015), 70-78. https://doi.org/10.1123/jab.23.1.70

Batmanabane, M., y Malathi, S. (1985). Movements at the Carpometacarpal and Metacarpophalangeal Joints of the Hand and Their Effect on the Dimensions of the Articular Ends of the Metacarpal Bones. 110, 102-110.

Bonilla Barrero, I. D., Pavajeau Escorcía, A. F., y Pérez Barbosa, W. A. (2019). Diseño y desarrollo de un dedo indice accionado por tendones.

Cheng, P., y Pearcy, M. (1999). A threedimensional definition for the flexion / extension and abduction / adduction angles. 37, 440-444.

Cheron, G., Petit, G., Cheron, J., Leroy, A., Cebolla, A., Cevallos, C., Dan, B. (2016). Brain oscillations in sport: Toward EEG biomarkers of performance. Frontiers in Psychology, 7, 246. https://doi.org/10.3389/fpsyg.2016.00246
Coert, J. H., van Dijke, G. A. H., Hovius, S. E. R., Snijders, C. J., y Meek, M. F. (2003). Quantifying thumb rotation during circumduction utilizing a video technique. Journal of Orthopaedic Research, 21(6), 11511155. 0266(03)00114-1

Conti, R., Meli, E., y Ridolfi, A. (2016). A novel kinematic architecture for portable hand exoskeletons. Mechatronics, 35, 192-207. https://doi.org/10.1016/j.mechatronics.2016.03. 002

Coupier, J., Hamoudi, S., Telese-Izzi, S., Feipel, V., Rooze, M., y Van Sint Jan, S. (2016). A novel method for in-vivo evaluation of finger kinematics including definition of healthy motion patterns. Clinical Biomechanics, 31, 4758.

https://doi.org/10.1016/j.clinbiomech.2015.10.0 02

Davis, R. B., Ounpuu, S., Tyburski, D., y Gage, J. R. (1991). A gait analysis data collection and reduction technique. Human Movement Science, 10(5), 575-587. https://doi.org/10.1016/01679457(91)90046-Z

Degeorges, R., Parasie, J., Mitton, D., Imbert, N., Goubier, N., y Lavaste, F. (2005). Threedimensional rotations of human three-joint fingers: an optoelectronic measurement . Preliminary results. 43-50. https://doi.org/10.1007/s00276-004-0277-4

Delp, S. L., Anderson, F. C., Arnold, A. S., Loan, P., Habib, A., John, C. T., Thelen, D. G. (2007). OpenSim: Open-source software to create and analyze dynamic simulations of movement. IEEE Transactions on Biomedical Engineering, 54(11), 1940-1950. https://doi.org/10.1109/TBME.2007.901024

Gonzalez, R. V., Buchanan, T. S., Delp, S. L. (1997). How muscle architecture and moment arms affect wrist flexion-extension moments. Journal of Biomechanics, 30, 705-712. https://doi.org/https://doi.org/10.1016/S00219290(97)00015-8

Gonzalez Cruz, F. C. (2019). Propuesta de vehículo autónomo para discapacitados en la región Piura. Universidad de Piura. 
González, L. D. (2019). Gimnasia artistica: Análisis biomecánico de la inversión adelante revisión sistemática. Acción Motriz, 22, 35-42.

Kapandji, A. I. (2006). Fisiología Articular. En $\begin{array}{llll}\text { Igarss } & 2014 & \text { (6a } & \text { ed.). }\end{array}$ https://doi.org/10.1007/s13398-014-0173-7.2

Lee, K. S., y Jung, M. C. (2015). Ergonomic evaluation of biomechanical hand function. Safety and Health at Work, 6(1), 9-17. https://doi.org/10.1016/j.shaw.2014.09.002

Mallon, W. J., Brown, H. R., y Nunley, J. A. (1991). Digital ranges of motion: Normal values in young adults. Journal of Hand Surgery, 16(5), 882-887. https://doi.org/10.1016/S03635023(10)80155-8

Mejía Navarrete, J. (2000). El muestreo en la investigación cualitativa. Investigaciones Sociales, pp. 165-180.

Nakamura, T., Yabe, Y., Horiuchi, Y., y Yamazaki, N. (1999). In vivo motion analysis of forearm rotation utilizing magnetic resonance imaging. 14, 315-320.

Nordin, M., Frankel, V. H., y Forssén, K. (2004). Biomecánica básica del sistema musculoesquelético (McGraw-Hill., Ed.). Interamericana.

Otzen, T., y Manterola, C. (2017). Técnicas de Muestreo sobre una Población a Estudio Sampling Techniques on a Population Study. Int. J. Morphol, 35(1), 227-232. https://doi.org/10.4067/S071795022017000100037

Tamayo Bullon, M. (2019). Estudio de la relación entre los pies y la organización de la postura del cuerpo para la comprensión corporal del desequilibrio en la danza contemporánea.

Wu, G., Van Der Helm, F. C. T., Veeger, H. E. J., Makhsous, M., Van Roy, P., Anglin, C., Buchholz, B. (2005). ISB recommendation on definitions of joint coordinate systems of various joints for the reporting of human joint motion Part II: Shoulder, elbow, wrist and hand. Journal of Biomechanics, 38(5), 981-992. https://doi.org/10.1016/j.jbiomech.2004.05.042 Zambrano, I., Cevallos, C., Obando, F.,
Jaramillo, M., Ortiz, D., Ojeda, D., Tobar Subía, L. M. (2018). Memorias del I Congreso Internacional de Bioingeniería y Sistemas Inteligentes de Rehabilitación. Diseño de interfaz mecatrónica para rehabilitación de mano y recuperación segura, 91-113.
RUIZ-CHICAIZA, Pablo \& CEVALlOS-BARRAGÁN, Carlos. Estudio cinemático de la mano para movimiento de oposición del dedo pulgar y movimientos de flexión y extensión de los dedos índice, medio, anular y meñique. Revista de Ingeniería Innovativa. 2019. 